\title{
Gemcitabine based combination chemotherapy in advanced pancreatic cancer-indirect comparison
}

\author{
Asma Sultana ${ }^{1}$, Paula Ghaneh ${ }^{1}$, David Cunningham ${ }^{2}$, Naureen Starling2, \\ John P Neoptolemos ${ }^{1}$ and Catrin Tudur Smith ${ }^{* 1,3}$
}

Address: ${ }^{1}$ CRUK Liverpool Cancer Trials Unit, Cancer Research Centre, 200 London Road, Liverpool, L3 9TA, UK, ${ }^{2}$ Department of Medicine, Royal Marsden Hospital, Downs Road, Sutton, Surrey SM2 5PT, UK and ${ }^{3}$ Centre for Medical Statistics and Health Evaluation, University of Liverpool, Shelley's Cottage, Brownlow Street, Liverpool, L69 3GS, UK

Email: Asma Sultana - asmasul@liv.ac.uk; Paula Ghaneh - P.Ghaneh@liverpool.ac.uk; David Cunningham - David.Cunningham@rmh.nhs.uk; Naureen Starling - Naureen.Starling@icr.ac.uk; John P Neoptolemos - j.p.neoptolemos@liv.ac.uk; Catrin Tudur Smith* - cat1@liverpool.ac.uk

* Corresponding author

Published: 8 July 2008

BMC Cancer 2008, 8:192 doi:10.1 186/147|-2407-8-192
Received: 14 October 2007

Accepted: 8 July 2008

This article is available from: http://www.biomedcentral.com/I47I-2407/8/192

(C) 2008 Sultana et al; licensee BioMed Central Ltd.

This is an Open Access article distributed under the terms of the Creative Commons Attribution License (http://creativecommons.org/licenses/by/2.0), which permits unrestricted use, distribution, and reproduction in any medium, provided the original work is properly cited.

\begin{abstract}
Background: Recent meta-analyses have found a survival advantage with gemcitabine based combinations over single agent gemcitabine in patients with advanced pancreatic cancer. There is paucity of evidence in the form of direct head-to-head randomised controlled trials to determine which combinations are to be preferred.
\end{abstract}

Method: Using the adjusted indirect comparison method proposed by Bucher et al, we have assessed randomised controlled trials of four gemcitabine based combinations namely gemcitabine plus a platinum compound or 5-fluorouracil or irinotecan or capecitabine.

Results: No particular combination was significantly superior to another, but the indirect evidence suggests some important trends.

Conclusion: The strongest trends on indirect comparison are towards favouring gemcitabine plus capecitabine or gemcitabine plus a platinum compound over gemcitabine plus irinotecan, and to a lesser degree, over gemcitabine plus 5 -fluorouracil.

\section{Background}

We have previously reported a systematic review and meta-analysis of 19 studies evaluating gemcitabine based combination chemotherapy compared to gemcitabine alone [1] in patients with locally advanced and metastatic pancreatic cancer. Overall survival was significantly better for gemcitabine based combination chemotherapy (14 trials 4060 patients HR 0.91 ; $95 \%$ CI 0.85 to 0.97 ) compared to single agent gemcitabine. A subgroup analysis was performed, dividing the selected studies into four categories defined by the addition to gemcitabine of plati- num agents or 5-fluorouracil (5FU) or irinotecan or capecitabine (Table 1). The subgroup analysis found evidence to suggest a survival advantage for gemcitabine combined with either a platinum agent (HR 0.85; 95\% CI 0.74 to 0.96 ) or capecitabine (HR $0.83 ; 95 \%$ CI 0.72 to 0.96 ), and insufficient evidence to support combinations of gemcitabine with either 5FU (HR 0.98; 95\% CI 0.86 to 1.11 ) or irinotecan (HR $1.01 ; 95 \% \mathrm{CI} 0.84$ to 1.22 ). These analyses provide estimates of the survival advantage for each combination compared to single agent gemcitabine but do not provide estimates of the survival advantage for 
Table I: List of included studies utilised in indirect comparison of gemcitabine based combination chemotherapy

\begin{tabular}{|c|c|c|}
\hline Comparison & Trial & Group (number randomised) \\
\hline \multirow[t]{3}{*}{$\begin{array}{l}\text { Gemcitabine versus gemcitabine plus } \\
\text { capecitabine }\end{array}$} & Cunningham 2005 (interim analyses) [12] & $\begin{array}{l}\text { Gemcitabine }(n=266) \\
\text { Gemcitabine combination }(n=267)\end{array}$ \\
\hline & $\begin{array}{l}\text { Hermann } 2005[13,14] \text { (analyses based on data from } \\
\text { abstract published in 2005, plus extra data provided by } \\
\text { trialist) }\end{array}$ & $\begin{array}{l}\text { Gemcitabine }(n=159) \\
\text { Gemcitabine combination }(n=160)\end{array}$ \\
\hline & Scheithauer $2003[15]$ & $\begin{array}{l}\text { Gemcitabine }(n=42) \\
\text { Gemcitabine combination }(n=4 I)\end{array}$ \\
\hline \multirow[t]{3}{*}{ Gemcitabine versus gemcitabine plus 5FU } & Berlin 2002 [8] & $\begin{array}{l}\text { Gemcitabine }(n=162) \\
\text { Gemcitabine combination }(n=160)\end{array}$ \\
\hline & Di Costanzo 2005 [10] & $\begin{array}{l}\text { Gemcitabine }(n=48) \\
\text { Gemcitabine combination }(n=43)\end{array}$ \\
\hline & Reiss 2005 [9] & $\begin{array}{l}\text { Gemcitabine }(n=236) \\
\text { Gemcitabine combination }(n=230)\end{array}$ \\
\hline \multirow[t]{3}{*}{$\begin{array}{l}\text { Gemcitabine versus gemcitabine plus } \\
\text { platinum compound }\end{array}$} & Heinemann 2006 [16] & $\begin{array}{l}\text { Gemcitabine }(n=99) \\
\text { Gemcitabine combination }(n=96)\end{array}$ \\
\hline & Louvet 2005 [17] & $\begin{array}{l}\text { Gemcitabine }(n=163) \\
\text { Gemcitabine combination }(n=163)\end{array}$ \\
\hline & Poplin 2006 [18] & $\begin{array}{l}\text { Gemcitabine }(n=280) \\
\text { Gemcitabine combination }(n=276)\end{array}$ \\
\hline \multirow[t]{2}{*}{$\begin{array}{l}\text { Gemcitabine versus gemcitabine plus } \\
\text { irinotecan }\end{array}$} & Rocha Lima 2004 [19] & $\begin{array}{l}\text { Gemcitabine }(n=180) \\
\text { Gemcitabine combination }(n=180)\end{array}$ \\
\hline & $\begin{array}{l}\text { Stathopoulos } 2005 \text { [20,2I] (analyses based on data from } \\
\text { abstract published in 2005, plus extra data provided by } \\
\text { trialist) }\end{array}$ & $\begin{array}{l}\text { Gemcitabine }(n=69) \\
\text { Gemcitabine combination }(n=57)\end{array}$ \\
\hline
\end{tabular}

each combination compared against another. To date, there is only one phase II randomised controlled trial $[2,3]$ which directly compared different gemcitabine combinations in a head-to-head comparison. This was a small study that directly compared only two gemcitabine based combination chemotherapy regimens (gemcitabine plus capecitabine versus gemcitabine plus oxaliplatin). In view of the paucity of data directly comparing alternative gemcitabine based combinations, we have attempted to answer, for the first time, as to which combinations of gemcitabine show more promise, an important clinical question which no previous meta-analyses have addressed.

\section{Methods}

We searched for direct comparisons of gemcitabine combinations, as well as used adjusted indirect comparisons to evaluate the treatment effect across studies [3,4] although this was not specified a priori. Illustration of how the indirect comparison was obtained is given in the following example. Suppose an intervention A was compared against another intervention $C$ in a randomised controlled trial or meta-analysis, and likewise another study (or meta-analysis) compared intervention B with intervention C. Adjusted indirect comparison of treatments A versus B was obtained as follows:

(1) The log hazard ratio of the adjusted indirect comparison for intervention A versus B was calculated using the following formula:

$$
\log \mathrm{HR}_{\mathrm{AB}}=\log \mathrm{HR}_{\mathrm{AC}}-\log \mathrm{HR}_{\mathrm{BC}}
$$

where $\log \mathrm{HR}_{\mathrm{AC}}$ was the log hazard ratio for the direct comparison of intervention $A$ versus $C$ and $\log H R_{B C}$ was the log hazard ratio for the direct comparison of intervention $\mathrm{B}$ versus $\mathrm{C}$.

(2) The standard error for the log hazard ratio was obtained using the calculation:

$$
\mathrm{SE}\left(\log \mathrm{HR}_{\mathrm{AB}}\right)=\sqrt{\mathrm{SE}\left(\log \mathrm{HR}_{\mathrm{AC}}\right)^{2}+\mathrm{SE}\left(\log \mathrm{HR}_{\mathrm{BC}}\right)^{2}}
$$

where $\mathrm{SE}\left(\log \mathrm{HR}_{\mathrm{AC}}\right)$ was the standard error of the log hazard ratio for the direct comparison of intervention A versus $C$ and $\mathrm{SE}\left(\log \mathrm{HR}_{\mathrm{BC}}\right)$ was the standard error of the log hazard ratio for the direct comparison of intervention $\mathrm{B}$ versus $C$.

The assumption of exchangeable treatment effects (treatment effect observed in trials comparing A versus $\mathrm{C}$ is assumed to be the treatment effect that would have been observed in those trials comparing B versus $C$ if treatment A had been included in those trials and vice versa) across comparisons was evaluated by assessing heterogeneity across trials within each comparison and assessing comparability (methodological and clinical characteristics) of all trials contributing to the indirect comparison. 


\section{Results}

Adjusted indirect comparisons were computed for the following comparisons:

1. Gemcitabine plus a platinum agent versus gemcitabine plus 5FU (GemPlat versus Gem5FU)

2. Gemcitabine plus a platinum agent versus gemcitabine plus capecitabine (GemPlat versus GemCap)

3. Gemcitabine plus a platinum agent versus gemcitabine plus irinotecan (GemPlat versus GemIrino)

4. Gemcitabine plus 5FU agent versus gemcitabine plus capecitabine (Gem5FU versus GemCap)

5. Gemcitabine plus 5FU agent versus gemcitabine plus irinotecan (Gem5FU versus GemIrino).

6. Gemcitabine plus capecitabine versus gemcitabine plus irinotecan (GemCap versus GemIrino)

We did not find any one combination to be significantly superior to others (Fig 1), but the indirect evidence suggests some important trends, of which the strongest are towards favouring gemcitabine plus capecitabine or gemcitabine plus a platinum compound over gemcitabine plus irinotecan (GemCap versus GemIrino HR 0.82, 95\% CI 0.65 to 1.04; GemPlat versus GemIrino HR 0.84, 95\% CI 0.67 to 1.06 ). Some advantage, to a lesser degree, was suggested for these two combinations over gemcitabine plus 5FU (Gem5FU versus GemCap HR 1.17, 95\% CI 0.94 to 1.46; GemPlat versus Gem5FU HR 0.88, 95\% CI 0.70 to 1.09 ). There was no evidence for heterogeneity within the direct comparisons and no obvious inconsistency with the assumption of exchangeable treatment effects.

Overall survival data was extracted from the single phase II randomised trial to allow estimation of a HR (95\% CI) [5] for the direct comparison between GemOx and GemCap. There was no significant difference in overall survival between the two combinations (HR 0.81, 95\% CI 0.56 to 1.18) on direct head-to-head comparison [2] as well as on computing this combining the results of both the direct and indirect comparisons (HR 0.94, 95\% CI 0.79 to 1.12) using random effects analysis.

\section{Discussion}

In a situation wherein two drugs A and B have, in randomised controlled trials, shown to be effective in comparison to a placebo or common standard, but direct comparison between $\mathrm{A}$ and $\mathrm{B}$ is not available, indirect comparison can be used [4]. The use of a simple indirect comparison has the limitation that the difference detected may not be a true difference, but instead be attributable to variations in patient characteristics and other prognostic factors in the different trials. An adjusted indirect comparison method was proposed by Bucher et al, and this method maintains the randomisation of the originally assigned patients while calculating the magnitude of the treatment effect.

Song et al assessed the comparability of indirect with the direct head to head comparison in the setting of clinical

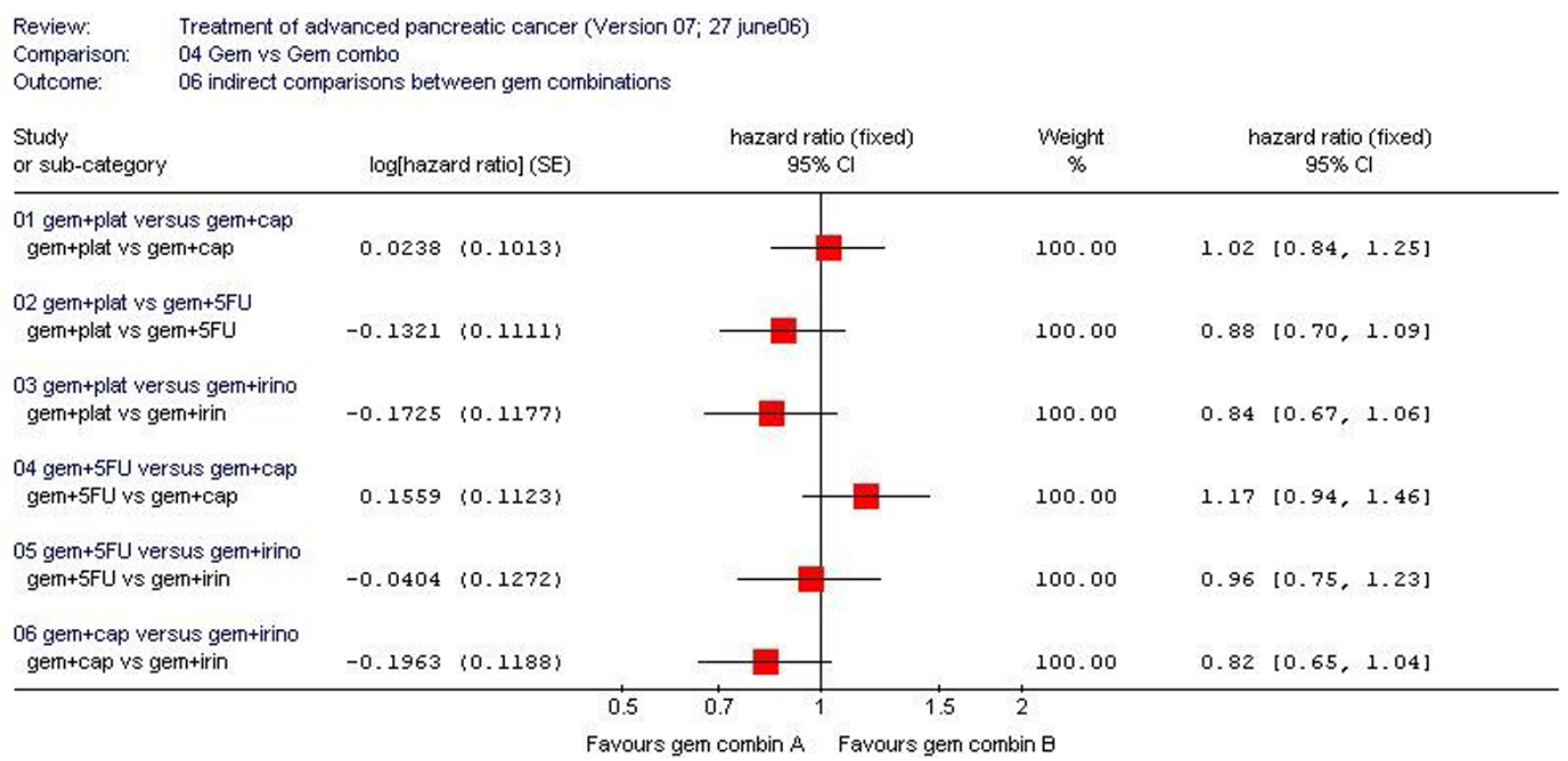

Figure I

Indirect comparison between different gemcitabine-based combination chemotherapy regimens. 
trials dealing with antimicrobial prophylaxis in colorectal cancer, and later using a sample of 44 comparisons from 28 systematic reviews $[3,6]$. Compared with direct estimates, the adjusted indirect estimates were less likely to be statistically significant. Adjusted indirect comparisons usually but not always agree with the results of head to head randomised trials. When there is no or insufficient direct evidence from randomised trials, the adjusted indirect comparison can provide useful or supplementary information on the relative efficacy of competing interventions. The results of adjusted indirect comparisons should be interpreted with caution however and the internal and external validity of the trials involved examined carefully, to investigate potential causes of discrepancy.

This adjusted indirect comparison of gemcitabine-based combination chemotherapy is very important clinically as there is paucity of evidence comparing these combinations and this is the first time hazard ratios have ever been calculated for these pairwise comparisons. The only randomised trial comparing gemcitabine combinations was a phase II multicentre study that compared capecitabine plus oxaliplatin(CapOx) versus capecitabine plus gemcitabine (CapGem) versus gemcitabine plus oxaliplatin (mGemOx) [2]. There was no significant difference in the primary end point of progression free survival [median progression free survival time $(\mathrm{p}=0.56)$ and progression free survival rates $(\mathrm{p}=0.67)]$ and overall survival $(\mathrm{Gem}$ Cap versus GemOx HR 0.81; 95\% CI 0.56-1.18). Grade 3/ 4 haematological toxicities were seen more often in the gemcitabine containing arms.

Although indirect evidence may not be as reliable as evidence from a randomised head to head comparison, these analyses show some interesting trends that could be used to direct future research priorities. The assumption of exchangeable treatment effects would seem reasonable for these comparisons which add strength to the clinical interpretation and conclusions. In particular, trends suggest that gemcitabine plus irinotecan may be the least effective of the combinations examined. The lack of significant differences on indirect comparison is probably due to the already highlighted observation that this method tends to yield results that are less statistically significant than in a direct comparison [6]. Indeed, it can be shown that one directly randomised trial is as precise as an indirect comparison based on four randomised trials of the same size.

A note-worthy observation on indirect comparison was that overall survival with gemcitabine combined with the fluoropyrimidine 5FU was inferior (though not statistically significant) to gemcitabine plus another fluoropyrimidine capecitabine (HR 1.17). A likely explanation is that capecitabine, an oral prodrug of 5FU, has the advan- tage of an element of tumour targeting, leading to enhanced selectivity and better tolerability [7]. The higher levels of thymidine phorphorylase (the final requisite enzyme for conversion of capecitabine to 5FU) observed in tumours compared to normal tissue may account for the improved targeting. Another possibility is the mode of delivery of 5FU versus capecitabine. The 5FU trials have involved bolus 5FU schedule [8] or 24 hour infusion [9], with the exception of one trial where 5FU was given by continuous infusion [10]. In contrast, the administration of capecitabine is more analogous to the delivery of 5FU by continuous protracted venous infusion, with the added ease of oral administration.

In the light of level I evidence demonstrating that gemcitabine based combinations have a modest survival advantage over single agent gemcitabine, the current study indicates which combinations may be more efficacious. The findings of our original meta-analyses, as well as the trends observed on our adjusted indirect comparisons support the use of gemcitabine in combination with either capecitabine or a platinum compound in clinical practice. Future randomised controlled trials will now likely to be centred on the exploitation of novel targets or biology (such as Telovac) [11] in this chemo-resistant cancer, probably on a cytotoxic backbone of a gemcitabine combination.

\section{Conclusion}

Adjusted indirect comparison of randomised controlled trials examining gemcitabine in combination with capecitabine, platinum based compounds, 5FU and irinotecan reveal trends towards favouring gemcitabine plus capecitabine or gemcitabine plus a platinum compound over gemcitabine plus irinotecan and to a lesser degree, over gemcitabine plus 5-fluorouracil. Future trials will now likely to be centred on the exploitation of novel targets or biology, probably on a cytotoxic backbone of a gemcitabine combination.

\section{Abbreviations}

CI: Confidence interval; GemCap: Gemcitabine plus capecitabine; GemIrino: Gemcitabine plus irinotecan; GemPlat: Gemcitabine plus platinum compound; HR: Hazard ratio.

\section{Competing interests}

The authors declare that they have no competing interests.

\section{Authors' contributions}

AS, CTS, JPN and PG were involved in the study design, data collection and analysis and manuscript write-up. DC and NS were involved in drafting the manuscript. All authors have read and approved the final manuscript. 


\section{Acknowledgements}

Cancer Research UK

\section{References}

I. Sultana A, Tudur Smith C, Cunningham D, Starling N, Neoptolemos J, Ghaneh P: Meta-Analyses of Chemotherapy for Locally Advanced and Metastatic Pancreatic Cancer. J Clin Oncol 2007, 25(18):2607-26I5.

2. Boeck S, Hoehler T, Seipelt G, Mahlberg R, Wein A, Hochhaus A, Boeck H, Schmid B, Kettner E, Stauch M, Lordick F, Ko Y, Geissler M, Schoppmeyer K, Kojouharoff G, Golf A, Neugebauer S, Heinemann V: Capecitabine plus oxaliplatin (CapOx) versus capecitabine plus gemcitabine (CapGem) versus gemcitabine plus oxaliplatin (mGemOx): final results of a multicentre randomised phase II trial in advanced pancreatic cancer. Ann Oncol 2008, 19:340-347.

3. Song F, Glenny A-M, Altman D: Indirect comparison in evaluating relative efficacy illustrated by antimicrobial prophylaxis in colorectal surgery. Control Clin Trials 2000, 21 (5):488-497.

4. Bucher H, Guyatt G, Griffith L, Walter S: The results of direct and indirect treatment comparisons in meta-analysis of randomised controlled trials. J Clin Epidemiol 1997, 50(6):683-69l.

5. Parmar M, Torri V, Stewart L: Extracting summary statistics to perform meta-analyses of the published literature for survival endpoints. Statistics in Medicine 1998, 17:28I5-2834.

6. Song F, Altman D, Glenny A-N, Deeks J: Validity of indirect comparison for estimating efficacy of competing interventions: emperical evidence from published meta-analyses. BMJ 2003, 326(7387):472-477.

7. Smith DB, Neoptolemos JP: Capecitabine in carcinoma of the pancreas. Expert Opin Pharmacother 2006, 7(12):1633-1639.

8. Berlin JD, Catalano P, Thomas JP, Kugler JW, Haller DG, Benson AB 3rd: Phase III study of gemcitabine in combination with fluorouracil versus gemcitabine alone in patients with advanced pancreatic carcinoma: Eastern Cooperative Oncology Group Trial E2297. J Clin Oncol 2002, 20(15):3270-3275.

9. Reiss H, Helm A, Niedergethmann M, Schmidt-Wolf I, Moik M, Hammer C, Zippel K, Weigang-Kohler K, Stauch M, Oettle H: A randomised, prospective, multicentre, phase III trial of gemcitabine, 5-Fluorouracil, folinic acid versus gemcitabine alone in patients with advanced pancreatic cancer. ASCO Annual Meeting: 2005 2005. abstract no. LBA4009

10. Di Costanzo F, Carlini P, Doni L, Massidda B, Mattioli R, lop A, Barletta E, Moscetti L, Recchia F, Tralongo P, Gasperoni S: Gemcitabine with or without continuous infusion 5-FU in advanced pancreatic cancer: a randomised phase II trial of the Italian oncology group for clinical research (GOIRC). $\mathrm{Br} J$ Cancer 2005, 93:185-189.

II. UK Clinical Research Network: TeloVac: A prospective, phase III, controlled, multicentre, randomised clinical trial comparing combination Gemcitabine and Capecitabine therapy with concurrent and sequential chemoimmunotherapy using a telomerase vaccine in locally advanced and metastatic pancreatic cancer. 2007 [http://pfsearch.ukcrn.org.uk/Stud yDetail.aspx? Topic $|\mathrm{D}=| \&$ Study $|\mathrm{D}=22| 0]$.

12. Cunningham D, Chau I, Stocken D, Davies C, Dunn J, Valle J, Smith D, Steward W, Harper P, Neoptolemos J: Phase III randomised comparison of gemcitabine versus gemcitabine plus capecitabine in patients with advanced pancreatic cancer. Eur J Can 2005, 4:. Abstract PSII

13. Hermann R, Bodoky G, Ruhstaller T, Glimelius B, Saletti P, Bajetta E, Schueller J, Bernhard J, Dietrich D, Scheithauer W: Gemcitabine plus capecitabine versus gemcitabine alone in locally advanced or metastatic pancreatic cancer. A randomised phase III study of the Swiss Group for Clinical Cancer Research (SAKK) and the Central European Cooperative Oncology Group (CECOG). 2005 ASCO Annual Meeting: 2005; Orlando, USA 2005. Abstract no. LBA40I0

14. Herrmann R, Bodoky G, Ruhstaller T, Glimelius B, Bajetta E, Schueller J, Saletti P, Bauer J, Figer A, Pestalozzi B, Kohne C, Mingrone W, Stemmer S, Tamas K, Kornek GV, Koeberle D, Cina S, Bernhard J, Dietrich D, Scheithauer W, Swiss Group for Clinical Cancer Research, Central European Cooperative Oncology Group: Gemcitabine plus capecitabine compared with gemcitabine alone in advanced pancreatic cancer: a randomised, multicenter, phase III trial of the Swiss Group for Clinical Cancer Research and the Central European Cooperative Oncology Group. J Clin Oncol 2007, 25(16):22 I 2-7.

15. Scheithauer W, Schull B, Ulrich-Pur H, Schmid K, Raderer M, Haider K, Kwasny W, Depisch D, Schneeweiss B, Lang F, Kornek GV: Biweekly high-dose gemcitabine alone or in combination with capecitabine in patients with metastastic pancreatic adenocarcinoma: a randomised phase II trial. Ann Oncol 2003, 14:97-104.

16. Heinemann V, Quietzsch D, Gieseler F, Gonnermann M, Schonekas $H$, Rost A, Neuhaus H, Haag C, Clemens M, Heinrich B, Vehling-Kaiser U, Fuchs M, Fleckenstein D, Geisierich W, Uthgennant D, Einsele $H$, Holstege A, Hinke A, Schalhorn A, Wilkowski R: Randomised phase III trial of gemcitabine plus cisplatin compared with gemcitabine alone in advanced pancreatic cancer. J Clin Oncol 2006, 24(24):3946-3952.

17. Louvet C, Labianca R, Hammel P, Lledo G, Zampino M, Andre T, Zaniboni A, Ducreux M, Aitini E, Taieb J, Faroux R, Lepere C, de Gramont A: Gemcitabine in combination with oxaliplatin compared with gemcitabine alone in locally advanced or metastatic pancreatic cancer: Results of a GERCOR and GISCAD phase III trial. J Clin Oncol 2005, 23(I 5):3509-35I6.

18. Poplin E, Levy D, Berlin J, Rothenberg M, Cella D, Mitchell E, Alberts $S$, Benson A iii: Phase III trial of gemcitabine (30-minute infusion) versus gemcitabine (fixed-dose rate infusion) versus gemcitabine + oxaliplatin in patients with advanced pancreatic cancer (E620I). J Clin Oncol 2006, 24(No I8S June 20 Suppl):. LBA4004

19. Rocha Lima C, Green M, Rotche R, Miller W, Jeffrey G, Cisar L, Morganti $A$, Orlando N, Gruia G, Miller L: Irinotecan plus gemcitabine results in no survival advantage compared with gemcitabine monotherapy in patients with locally advanced or metastatic pancreatic cancer despite increased tumor response rate. J Clin Oncol 2004, 22( ( 8):3776-3783.

20. Stathopoulos G, Aravantinos G, Syrigos K, Kalbakis K, Karvounis N, Papakotoulas P, Boukovinas J, Potamianou A, Polyzos A, Christophillakis C, Georgoulias V: A randomised phase III study of irinotecan/gemcitabine combination versus gemcitabine in patients with advanced/metastatic pancreatic cancer. 2005 ASCO Annual Meeting: 2005; Orlando, USA 2005. Abstract no. 4I06

21. Stathopoulos G, Syrigos K, Aravantinos G, Polyzos A, Papakotoulas P, Fountzilas P, Potamianou A, Ziras N, Boukovinas J, Varthalitis J, Androulakis N, Kotsakis A, Samonis G, Georgoulias V: A multicentre phase III trial comparing irinotecan-gemcitabine with gemcitabine monotherapy as first-line treatment in patients with locally advanced or metastatic pancreatic cancer. $\mathrm{Br} J$ Cancer 2006, 95(5):587-592.

\section{Pre-publication history}

The pre-publication history for this paper can be accessed here:

http://www.biomedcentral.com/1471-2407/8/192/pre pub

Publish with Bio Med Central and every scientist can read your work free of charge

"BioMed Central will be the most significant development for disseminating the results of biomedical research in our lifetime. "

Sir Paul Nurse, Cancer Research UK

Your research papers will be:

- available free of charge to the entire biomedical community

- peer reviewed and published immediately upon acceptance

- cited in PubMed and archived on PubMed Central

- yours - you keep the copyright 